\title{
Niger-Congo Language
}

National Cancer Institute

\section{Source}

National Cancer Institute. Niger-Congo Language. NCI Thesaurus. Code C161852.

A large family of languages spoken in Africa, predominantly in sub-Saharan Africa. 